\title{
AP-IO: Asynchronous Pipeline I/O for Hiding Periodic Output Cost in CFD Simulation
}

\author{
Ren Xiaoguang and Xu Xinhai \\ State Key Laboratory of High Performance Computing, National University of Defense Technology, Changsha, Hunan 410073, China
}

Correspondence should be addressed to Ren Xiaoguang; hbszrxg@gmail.com

Received 26 December 2013; Accepted 2 March 2014; Published 3 April 2014

Academic Editors: S. K. Bhatia and P. Muller

Copyright (C) 2014 R. Xiaoguang and X. Xinhai. This is an open access article distributed under the Creative Commons Attribution License, which permits unrestricted use, distribution, and reproduction in any medium, provided the original work is properly cited.

\begin{abstract}
Computational fluid dynamics (CFD) simulation often needs to periodically output intermediate results to files in the form of snapshots for visualization or restart, which seriously impacts the performance. In this paper, we present asynchronous pipeline $\mathrm{I} / \mathrm{O}(A P-I O)$ optimization scheme for the periodically snapshot output on the basis of asynchronous I/O and CFD application characteristics. In AP-IO, dedicated background I/O processes or threads are in charge of handling the file write in pipeline mode, therefore the write overhead can be hidden with more calculation than classic asynchronous $\mathrm{I} / \mathrm{O}$. We design the framework of $A P-I O$ and implement it in OpenFOAM, providing CFD users with a user-friendly interface. Experimental results on the Tianhe2 supercomputer demonstrate that $A P-I O$ can achieve a good optimization effect for the periodical snapshot output in CFD application, and the effect is especially better for massively parallel CFD simulations, which can reduce the total execution time up to about $40 \%$.
\end{abstract}

\section{Introduction}

Currently, computational fluid dynamics (CFD) is increasingly applied in science and engineering with the continuous development of computer technology, especially the evergrowing computing power of supercomputers [1-3]. In order to meet the needs of visualization or restart, CFD simulation has to periodically output intermediate results to files in the form of snapshot.

Unfortunately, this kind of periodic snapshot output has gradually become a performance bottleneck in CFD simulations on massively parallel system. In addition, according to some experimental data [4-6], the periodic large volume snapshot file output can take more than $80 \%$ time of the whole CFD simulation process in extreme case. On the one hand, the growth rate of $\mathrm{I} / \mathrm{O}$ speed is far behind the growth rate of computing capacity $[7,8]$, which makes I/O become a bottleneck; on the other hand, the requirement of all-refined numerical simulation promotes the mesh scale of many CFD applications to million scale, resulting in the snapshot file tremendous, that is, Gigabytes or even Terabytes $[9,10]$.
Therefore, it is urgent to optimize the periodic large volume snapshot file output in massively parallel CFD simulations.

Asynchronous I/O is a widely used I/O optimization technique, in which dedicated I/O processes/threads are used to handle the file write operations, and the I/O overhead is hidden by overlapping I/O operations with calculation operations $[11,12]$. The key of the asynchronous I/O optimization is to get enough calculation operations to hide the I/O overhead on the premise of data consistency; that is, for any set of data, its asynchronous write operation should be finished before the next update operation.

A CFD simulation, as one kind of typical computeintensive application, has an immense potential to use asynchronous I/O technology to optimize large-volume file output. However, the snapshot output in traditional CFD simulation always happens at the end of a timestep, which is close to the update operation of the same data at the beginning of next timestep. Thus, there is no enough time for asynchronous I/O to hide the file write overhead.

By analyzing the simulation procedure of CFD applications, which is based on the time step iterations, we find 
that each timestep's snapshot generally consists of multiple physical data arrays called field, and these fields are updated at different time. This potentially allows to output these fields asynchronously in pipeline mode. In this paper, we propose $A P-I O$, a new asynchronous pipeline I/O optimization method for periodic large-volume snapshot output in massively parallel CFD applications. Instead of outputting snapshot's multiple fields together at the end of the time step, AP-IO asynchronously outputs these different fields in a pipeline mode. below.

The main contributions of this paper are summarized

(i) We introduce $A P-I O$, an asynchronous pipeline $\mathrm{I} / \mathrm{O}$ method, to solve the I/O performance bottleneck problem of periodic large volume snapshot output in CFD applications.

(ii) We propose an application level calculation segments scheduling method, which schedules the order of the calculation segments of different fields, to obtain more hidden time for AP-IO.

(iii) We implement the user-level $A P-I O$ framework in the open source CFD software OpenFOAM with the Pthread and compiler-directed instruction techniques.

(iv) We show by experiments on Tianhe-2 supercomputer with three typical CFD application cases that $A P$ $I O$ optimization method can significantly enhance the performance of periodic large-volume snapshot output in CFD applications. Specifically, the file write overhead can be reduced $60 \%$ on average, and the total execution time can be reduced $18 \%$ on average.

The rest of the paper is organized as follows. Section 2 introduces the motivation of our work. Section 3 introduces the basic idea of $A P-I O$. Section 4 describes the framework of $A P-I O$, including the programming model, the compilerdirected foundation framework, and the calculation segments scheduling optimization. Section 5 introduces the implementation of AP-IO in OpenFOAM software. Section 6 describes our evaluation methodology and demonstrates the superiority of $A P-I O$ by $3 \mathrm{CFD}$ applications. Finally, Section 7 concludes the paper.

\section{Motivation}

2.1. Snapshot Output in CFD. In a typical CFD application, on the one hand, the simulation procedure is discretized into several timesteps, and each timestep is denoted with $\Delta t$. It is notable that different timesteps are simulated in the same computing mode, but with different initial condition value. On the other hand, the simulation region is discretized into a mesh system, and the physical property in the simulation region is represented by a filed, that is, a data array with the same size of the mesh. As shown in Figure 1(a), each timestep is usually simulated by computing multiple fields one by one. The set of these fields, namely, snapshot, is the intermediate simulation result of the timestep.

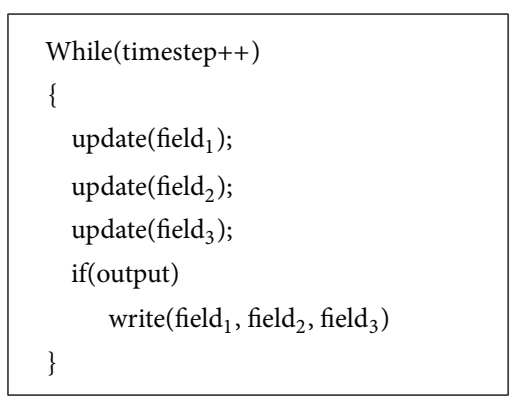

(a) Example code of Snapshot output

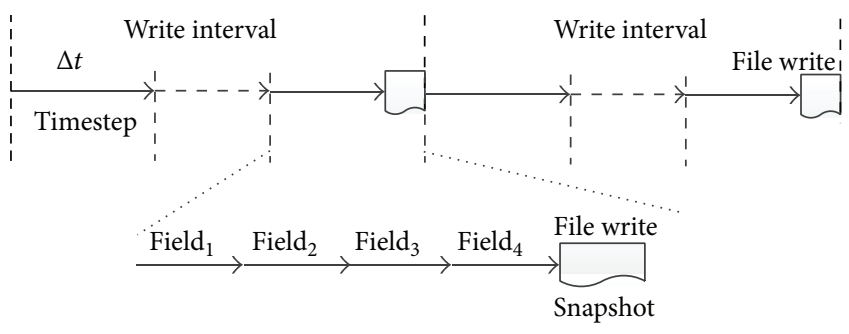

(b) The snapshot output mode

FIGURE 1: Example code and snapshot output mode of a typical CFD application.

For the purpose of visualization or restart, a CFD simulation procedure needs to periodically output the snapshot to files on the disk. As shown in Figure 1(b), the snapshot is outputted at the end of write interval, which is consisted of fixed number of timesteps.

Nowadays, the mesh size of massively parallel CFD applications reaches million level, and the volume of a snapshot often scales to Gigabyte level. Unfortunately, outputting such large snapshot on HPC system seriously affects the performance of the whole CFD simulation. The reasons are as follows: (a) multiple users or multiple processes competing for shared I/O channel; (b) with the scale of HPC increasing, the performance of computing capability improves more quickly than that of $\mathrm{I} / \mathrm{O}$; (c) the redundant data produced by the parallel task partitioning makes the volume of snapshot rise. All these influences will be discussed in detail in Section 6.

According to our experiments as well as in related documents, the file write overhead consumes $20 \%$ of the whole CFD simulation time on average, which can reach even more than $80 \%$ in extreme circumstances [4-6]. Thus, it is critically important to optimize the periodical large volume snapshot output in massively parallel CFD applications.

2.2. Traditional Optimization for File Write. I/O performance optimizations mainly performed in two ways: (a) reducing I/O overhead and (b) hiding I/O overhead.

Buffer mechanism [8], which reduces I/O overhead through buffer caching, is the most universal technology used for reducing $\mathrm{I} / \mathrm{O}$ overhead in the modern computer system. However, the buffer mechanism is restrained by buffer capacity and difficult to be applied in large-volume file output. In most modern computer systems, the buffer size 


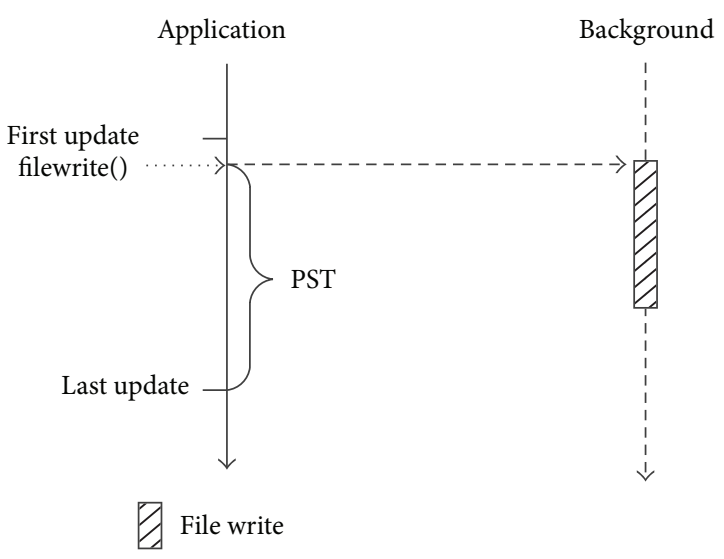

FIGURE 2: Asynchronous I/O for large volume file.

is less than 1 Megabyte, then the buffer mechanism cannot optimize the CFD snapshot output, which is usually more than hundreds of Megabytes.

Asynchronous I/O is another optimization technology used for hiding I/O overhead. The core idea of asynchronous $\mathrm{I} / \mathrm{O}$ is to use dedicated routines (processes/threads) in background to perform I/O operation, which will hide the file write overhead with calculation operations [13]. As shown in Figure 2, the asynchronous write operations in the background overlap with calculation operations and the file write overhead is hidden. To guarantee the data consistency, for any set of data, the background asynchronous write operation must be finished before the next update operation of the same data.

So, for asynchronous $\mathrm{I} / \mathrm{O}$, it is critical that there are enough calculation operations to hide the file write overhead. As a matter of convenience, we define potential shield time (PST) as the calculation time used to hide the write overhead. To a data set, if the output operation is closed to the next update operation of the same data, the PST will not be sufficient to hide the file write overhead.

\section{Basic Idea}

3.1. The Basic Idea of AP-IO. In the parallel CFD simulation, there are multiple simulation processes which we call as compute processes. As shown in Figure 3(a), it is an example of the asynchronous I/O method applied for snapshot output in the CFD simulation. For each compute process, there is a corresponding dedicated write service routine (process/thread) to deal with the file write requests. When a compute process needs to do a write operation, it sends a relevant write request to its corresponding write service routine, which handles the request in background.

A prominent problem of the above traditional asynchronous I/O for the snapshot output in the CFD simulation is the shortage of PST. The outputs of all the fields of a snapshot are concentrated at the end of the timestep, which is close to their update operations in the next timestep. In asynchronous $\mathrm{I} / \mathrm{O}$, to guarantee the data consistency, these fields' update operations in the next timestep cannot be performed until the asynchronous write operations in the current timestep are completed. This situation makes it clear that there is no PST for the asynchronous snapshot output.

In order to solve this problem, we proposed the AP-IO method. AP-IO method mainly originates from the updating characteristic of the fields of the snapshot. In general, as shown in Figure 4(a), a field may be updated for several times in a timestep, We call an updating procedure as calculation segment, and the calculation segments of a field are stagger with other fields' calculation segments. For a field in a single timestep, we define the First Update (FU) time and the Last Update $(L U)$ time. The $F U$ time of a field is the starting point of the field's first calculation segment in a timestep, and the $L U$ time is the end point of the field's last calculation segment in a timestep.

In $A P-I O$, as shown in Figure 3(b), the fields of the snapshot are not outputted together at the end of the time step. On the contrary, for each field, its output follows its $L U$ time in the current timestep. To guarantee the data consistency, the field's output operation should be finished before its FU time in the next time step. So, the PST of a field is the time between the $L U$ time in the current timestep and the $F U$ time in the next timestep. Compared with the centralized output at the end of timestep, AP-IO gets more PST, and the field's output operation will be overlap with other fields' calculation operations.

3.2. Analysis of PST. Providing more PST is the key advantage of $A P-I O$, and we will analyze the characteristics of PST in CFD simulation in this section.

The distribution of a field's calculation segments in a single timestep has a direct influence on the length of its PST in the AP-IO mode. Considering the CFD simulation process shown in Figure 4(a), the field ${ }_{0}$ 's calculation operations go across the entire timestep. field ${ }_{0}$ 's $L U$ time in the current timestep is overlapped with its $F U$ time in the next timestep, resulting in the fact that there is no PST for field 0 . To obtain more PST for field $_{0}$, as shown in Figure 4(b), we exchange field $_{1}$ 's first calculation segment with field , $^{\text {'s first }}$ calculation segment. Now, field ${ }_{0}$ can get a PST PST 0 as shown in Figure 4(b).

In order to increase a fields' PST, we need to improve the distribution of fields' calculation segments in the snapshot. A calculation segments scheduling optimization method, aiming at improving the distribution of fields, is proposed in this paper, which will be described in Section 4.3.

\section{Framework}

We design the user-level framework of $A P$-IO by combing the compiler-directed technology with the AP-IO library, which is convenient for CFD users to carry out the $A P-I O$ optimization in their CFD applications.

4.1. Programming Model. We define two compiler directives for the AP-IO optimization, and their semantics are listed as follows. 


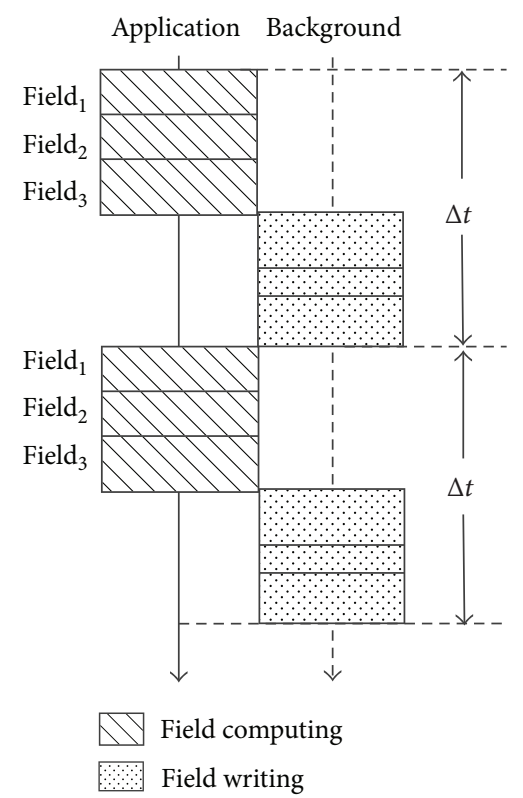

(a) Asynchronous IO for snapshot output

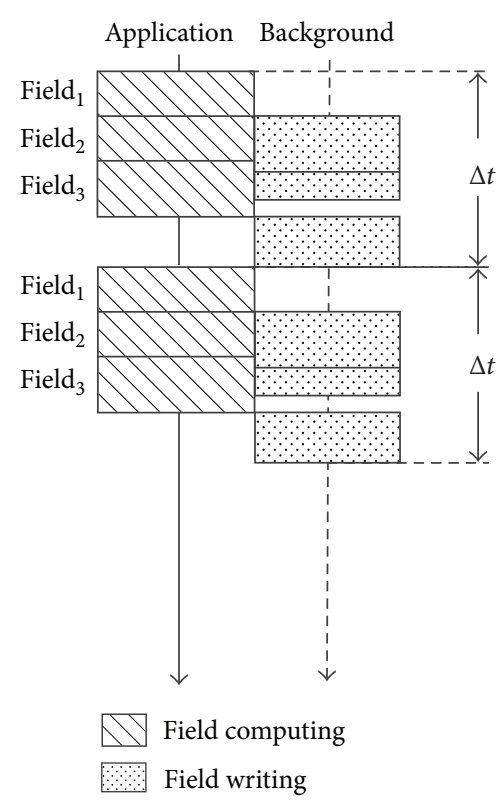

(b) AP-IO for snapshot output

FIGURe 3: Asynchronous IO versus AP-IO for CFD snapshot output.

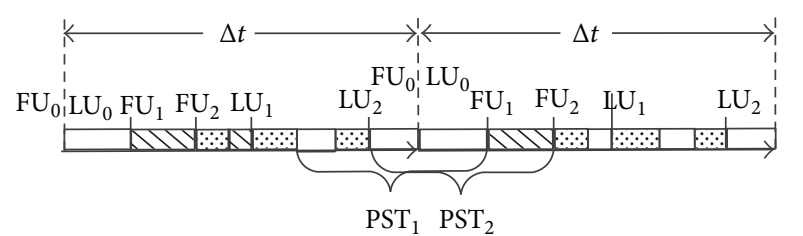

Field $_{0}$ computing $\quad \because$ Field $_{2}$ computing

$\triangle$ Field $_{1}$ computing

(a)

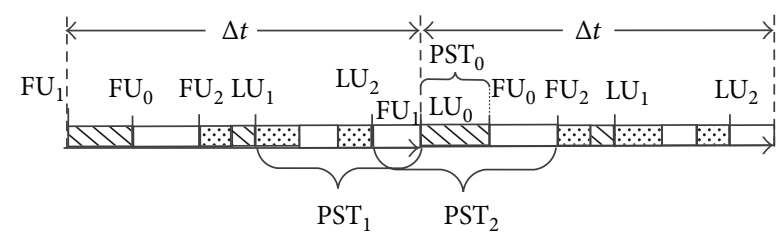

Field $_{0}$ computing $\quad \because$ Field $_{2}$ computing

$\triangle$ Field $_{1}$ computing

(b)

Figure 4: Example of PST.

(i) “\#FU field ${ }_{1}$ ": to explicitly identify field 1 's FU time, which means when the execution goes across this point, the asynchronous write of field 1 is no longer safe, and it is required to take a blocking wait to guarantee the finish of field, 's asynchronous write.

(ii) "\#LU field,": to explicitly identify field ${ }_{1}$ 's $L U$ time, which means that from this point on, the request of field $_{1}$ 's write can be sent to the background write service routine to perform asynchronous write.
We will illustrate the AP-IO programming model with an example. The left part of Figure 5 shows a typical CFD application code based on timestep iteration. The update procedure of field $_{1}$ in the code has three calculation segments: update 1(), update2(), update3(). If we use AP-IO to optimize the periodic snapshot output, we should add the compile directives in the corresponding position of the code for each field. Take field 1 as an example, we need to add compile directives "\#FU field ${ }_{1}$ " in front of update 1() and "\#LU field 1 " after update3(), respectively. These two compile directives explicitly define the PST of field ${ }_{1}$.

This compiler-directive based programming model makes minimum modification of the original CFD application code and enables the CFD application developers to optimize with the $A P-I O$ method at minimum cost. It is notable that the compiler directives can be automatically inserted through compile techniques. However, it is not the focus of this paper.

4.2. Framework of AP-IO. Figure 5 presents our AP-IO framework, the core components which include both the $A P$ $I O$ compiler and $A P-I O$ library. The $A P-I O$ compiler is a compile directives based source-to-source compiler. The CFD application code in the left part of Figure 5 is compiled into the code including AP-IO library calls which is shown in the right part of Figure 5.

The AP-IO library provides semantic interfaces support for the application code generated by the AP-IO compiler. As shown in Figure 5, after preprocessing by the $A P-I O$ compiler, the compiler directives, "\#LU field ${ }_{1}$ " and "\#FU field $_{1}$ ", are, respectively, replaced with $A P-I O$ library calls: Iwrite (field ${ }_{1}$ ) and Iwait ( field $)_{1}$, whose semantic functions are, respectively, listed as follows. 


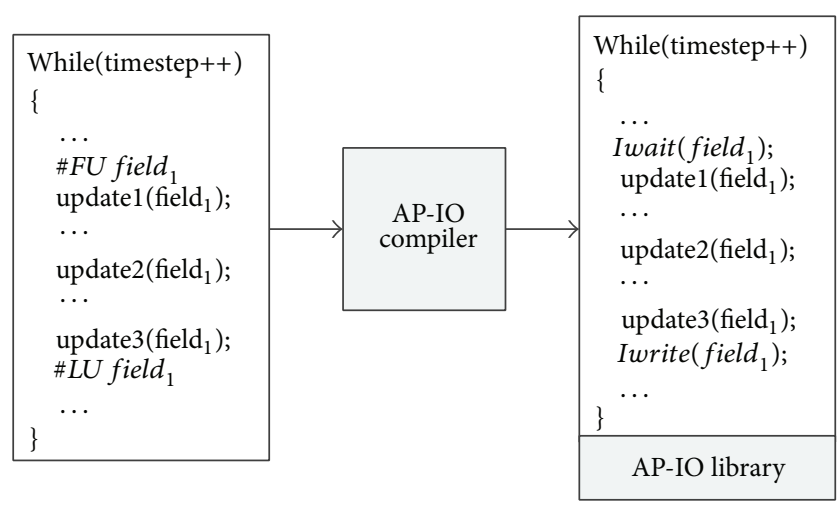

FIgURE 5: Framework of AP-IO.

(i) Iwrite (field 1 ): responsible for sending the field,'s write request to the background service routine. The function will return as soon as the request is successfully sent. Iwrite() semantics encapsulate three work: the send of asynchronous write request, FIFOmode queuing of write requests in the write service routine, background write, and other operations.

(ii) Iwait ( field $\left._{1}\right)$ : responsible for checking whether the corresponding field's asynchronous write operation has been finished. If it has been already finished, a success code will be returned. Otherwise, a blocking wait must be hold until the finish of the asynchronous write operation, to avoid updating the field whose write operation is underway.

4.3. Calculation Segments Scheduling Optimization. In order to gain the benefit of $A P-I O$, our AP-IO compiler can also help the developer to optimize the distribution of the calculation segments of each field in a single timestep. Achieving the most optimal scheduling solution is a NP-hard problem. Therefore, we design a heuristic algorithm for the scheduling of field's calculation segments in a single timestep, which is shown in Algorithm 1.

The core idea of our heuristic algorithm is to improve the distribution of each field's calculation segments, that is, reduce the intersection between different fields' calculation segments. First, the scheduling algorithm numbers the fields in a single timestep according to the order of each field's $F U$ time. During the scheduling, the algorithm moves the odd number field's segments as close as possible to its first calculation segment, and the even number field's segments as close as possible to its last calculation segment. "As possible" means maintaining the dependencies between the calculation segments, and moving the calculation segments as much as possible to the corresponding direction.

\section{Implementation of $\boldsymbol{A P}-\mathrm{IO}$}

We implement the AP-IO method in the open source CFD software OpenFOAM with the source-to-source compiler technology and the Pthread technology, which mainly includes two parts: the compiler support layer and the $A P-I O$ library.

5.1. Compiler Support Layer. With the source-to-source compile technology, the compiler support layer mainly includes two parts: transforming the compiler directives to $A P-I O$ library function calls and scheduling the fields' calculation segments in the origin OpenFOAM application code with the heuristic scheduling algorithm in Algorithm 1.

5.2. AP-IO Library. The AP-IO library mainly involves three parts: compute processes, write service threads, and write task queue, as shown in Figure 6.

5.2.1. Iwrite. First, a Pthread thread is created for each parallel simulation process (compute process) as the write service thread. A write task queue is shared by each compute process and its write service thread. When the compute process trigger the function call Iwrite(), a write request will be added to the write task queue. The write service thread in the background constantly scans the write task queue, and all the tasks in the queue are processed in FIFO mode.

A status variable writesstate is set for each field, and it has three states: WAIT_WRITE, WRITING, and WRITE_FINISHED, the meanings of them are listed as follows.

(i) WAIT_WRITE means that this field's write request in the write task queue is waiting to be handled by the write service thread.

(ii) WRITING means that this field's write request is being handled by the write service thread and this field is writing in the background now.

(iii) WRITE_FINISHED means that this field's write request had been finished, or there is no write request for this field.

For a field, the default status of writestate is WRITE_FINISHED. When the function Iwrite ( field $_{1}$ ) is called in compute process, the write request of field ${ }_{1}$ is added into the write task queue, and writestate of field 1 is set as WAIT_WRITE. When the write request begins to be handled by the write service thread, write_state of field 1 will be set as WRITING. Finally, the status will be set to WRITE_FINISHED when the write request is finished.

5.2.2. Iwait. To guarantee the data consistency, the compiledirective "\#FU field ${ }_{1}$ " explicitly specifies the opportunity of checking whether field ${ }_{1}$ 's write has been finished. The function call Iwait() in the compute process is in charge of the checking work. The checking work of Iwait() is performed by reading the status of write_state. If the status of write_state is WRITE_FINISHED, Iwait() returns with a success code. Otherwise, a blocking wait is launched and the waiting procedure will not finish until the status of write_state is updated to WRITE FINISHED. 
INPUT: the origin order set of calculation segments in a time step $F$, which includes $n$

calculation segments; the matrix of dependence relationship between calculation segments $D$;

OUTPUT: the new order set of calculation segments in a time step $F_{\text {new }}$;

(1) procedure $\operatorname{SCHEDULING}(F, D)$ // calculation segments scheduling procedure

(2) $\quad F_{\text {new }}=F$

(3) while $F_{\text {new }} \neq F_{\text {old }}$ do

(4) $\quad F_{\text {old }}=F_{\text {new }}$;

(5) for each $i \in[1, n]$ do

(6) if $\left(K\left(S_{i}\right) \% 2==0 \& \& D(i, i+1)==0 \& \& S_{i} \neq S_{k\left(S_{i}\right)}^{\text {first }}\right)$ then $/ / k\left(S_{i}\right)$ indicates

the field number which $S_{i}$ belongs to

(7) $\quad S_{i}$ exchange with $S_{i+1}$;

(8) end if

(9) if $\left(K\left(S_{i}\right) \% 2==1 \& \& D(i-1, i)==0 \& \& S_{i} \neq S_{k\left(S_{i}\right)}^{\text {last }}\right)$ then

(10) $\quad S_{i-1}$ exchange with $S_{i}$;

(11) end if

(12) end for

(13) if $\left(F_{\text {new }}==F_{\text {old }}\right)$ then

(14) break;

(15) end if

(16) end while

(17) end procedure

Algorithm 1: A heuristic algorithm for the scheduling of fields' calculation segments in a single timestep.

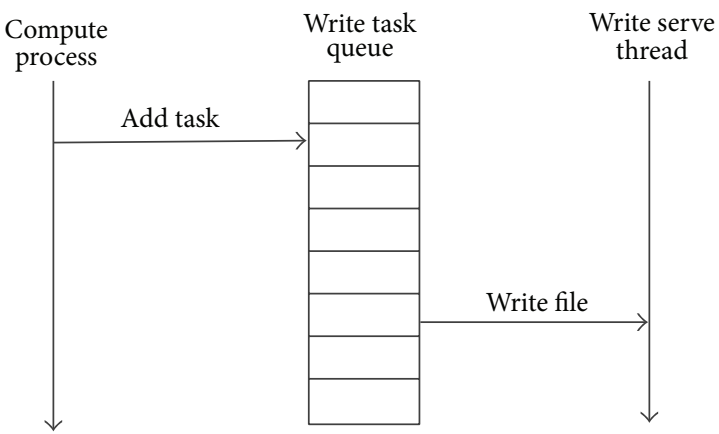

Figure 6: Implement of AP-IO.

5.2.3. Threading Optimizations. The above mechanism can basically perform the AP-IO optimization in the OpenFOAM platform, but there are two situations that should be taken into account for the CPU consumption, which influence the performance optimization.

The first situation is shown in Figure 7(a). In the Iwait() function call, if the status of writestate is WRITING or WAIT_WRITE, a blocking wait is needed. This blocking wait has two implementation ways. One is to constantly check the status of write_state with while iteration, which will seriously consume CPU resources, resulting in low efficiency. So, we take another more efficient way. In the procedure of the blocking wait, the compute process is suspended and sleeping to release CPU resources. Once the write service thread finishes the current write request, it awakens the sleeping compute process by sending a signal to it. Therefore, the compute process can greatly reduce the CPU resources consumption during the sleep time.
The second situation is shown in Figure 7(b), it is related to the write service thread's scan of the write task queue. When the queue is empty and no write request needs to be handled, to timely responds to the new write request, the write service thread has to pay close attention to the change of the write task queue. Similar to the first situation, once the write service thread finds that the write task queue is empty, it suspends itself to sleep and releases CPU resources. The compute process always tries to send an awake signal to the write service thread when a new write request arrives. If the write service thread is sleeping, it will be awakened at the time of new request's arrival.

The two suspend/awake mechanism can ensure that the compute process and the write service thread will not consume too many $\mathrm{CPU}$ resources, which guarantees the efficient execution of $A P-I O$ in CFD simulation.

\section{Experiment}

We demonstrate the superiority of our AP-IO method on the Tianhe-2 supercomputer with three typical CFD application cases. Section 6.1 introduces the computer platform Tianhe-2 used in the experiments. Section 6.2 discusses the benchmarks selected. Section 6.3 describes the experiment environment and the evaluation methodology. Section 6.4 presents and analyzes our results.

6.1. Platform. The Tianhe-2 supercomputer was built by China's National University of Defense Technology (NUDT), which is the world's fastest supercomputer with a 33.86 petaflop peak performance according to the TOP500 list in June 2013 [14]. Our experiments use a subsystem of Tianhe- 2 with 128 compute nodes as the test platform, and 


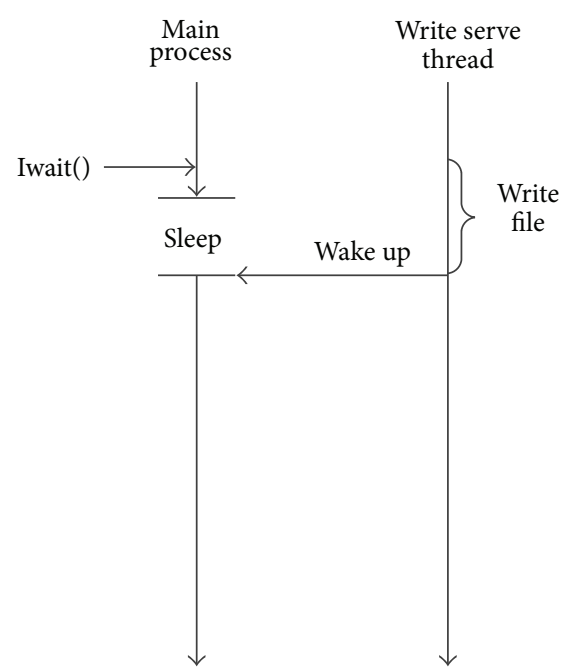

(a) Thread optimizations for Iwait()

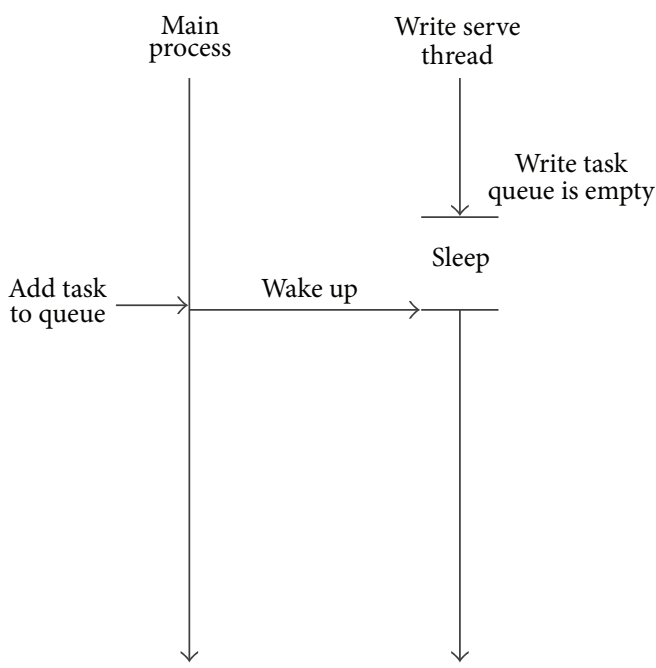

(b) Thread optimizations for write task queue

FIgURE 7: Threading optimizations for AP-IO.

each computing node comprises two Intel Ivy Bridge Xeon processors, three Xeon Phi chips (accelerator card), and 64 Gigabyte DRAM [15]. It should be pointed out that our experiments only use the processors, and do not use the accelerator card.

The whole I/O subsystem of Tianhe-2 has a disk array with a 12.4 Petabyte capacity, and all the nodes in the system share the disk array through a global interconnecting network. The interconnecting network uses the optoelectronics hybrid transport technology, connecting all the compute nodes through 576 connection ports with 13 large routers, and the transmission rate reaches $6.36 \mathrm{~GB} / \mathrm{s}$.

It is noticeable that although the subsystem we use is exclusive for us, the I/O system is globally shared with other users, which can lead to competition with other users and impact the precision of the experimental results. A detailed analysis of this problem will be described in Section 6.3.1.

6.2. Benchmark. To verify the superiority of $A P-I O$, we select three typical large-scale CFD applications as benchmarks. They are dambreak, cavity, and pitzDaily. The three benchmarks are briefly introduced as follows.

(i) Dambreak: dambreak is a simulation of dambreak based on the volume of fluid (VOF) method. The twophase flow solver interFoam in OpenFOAM is used.

(ii) Cavity: cavity is the lid-driven cavity flow simulation, using the incompressible flow solver icoFoam in OpenFOAM.

(iii) PitzDaily: pitzDaily is a large eddy simulation (LES) case. The solver used is pisoFoam together with the oneEqEddy eddy viscosity model.

Some important parameters of the three benchmarks are shown in Table 1. All the benchmarks are in 3D with the grid scale between $5 \mathrm{M}$ and $17 \mathrm{M}$. To verify the effect of the write interval to the performance of snapshot output, we tested five

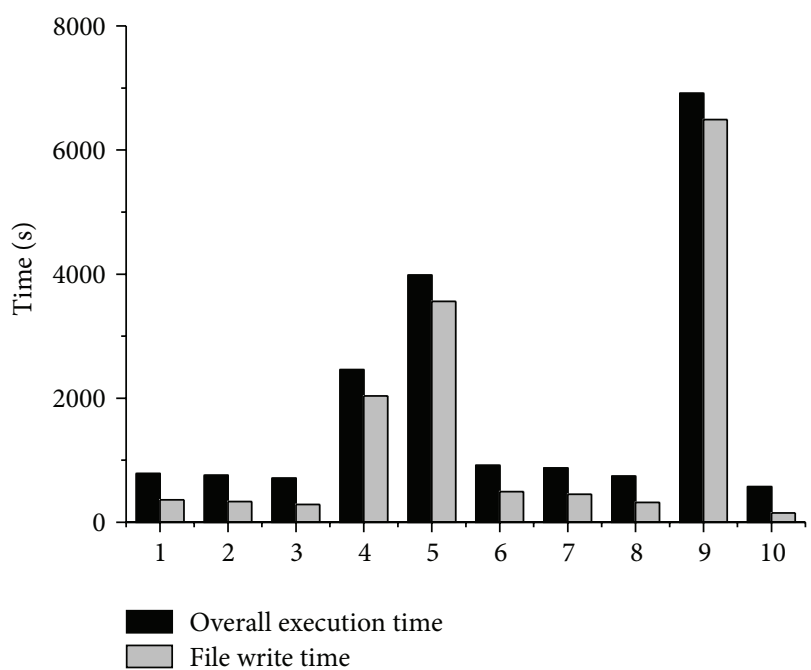

FIgUre 8: The execution time of cavity runs on Tianhe-2 for 10 times.

write interval conditions, including the none condition with no snapshot output. All the three benchmarks take Scotch as the decompose method for parallel grid partition.

\subsection{Experiment Design}

6.3.1. Experiment Environment Analysis. As mentioned in Section 6.1 that we use the I/O subsystem shared with other users, our experimental results are more or less random. To measure this randomness, we test the cavity benchmark for 10 times with 256 processors. The test is performed in a continuous time, during which the Tianhe-2 system is running hundreds of other users' tasks at the same time, and these tasks may also perform file write operations to the disk array. The execution times are shown in Figure 8. 
TABLE 1: The parameters of the three benchmark.

\begin{tabular}{lccc}
\hline & Dambreak & Cavity & PitzDaily \\
\hline Solver & interFoam & icoFoam & pisoFoam \\
Dimensionality & $3 \mathrm{D}$ & $3 \mathrm{D}$ & $3 \mathrm{D}$ \\
Number of cells & 6229504 & 15625000 & 16131636 \\
$\Delta t$ & $1.00 E-03$ & $1.00 E-04$ & $2.00 E-04$ \\
Number of time steps & 100 & 100 & 100 \\
Write interval (time Steps) & $1 / 2 / 4 / 10 /$ none & $1 / 2 / 4 / 10 /$ none & $1 / 2 / 4 / 10 /$ none \\
Number of fields & 5 & 8 & 8 \\
Decomposition method & Scotch & Scotch & Scotch \\
\hline
\end{tabular}

According to Figure 8, we can find that the execution times of the 4th, 5th, and 9th test are much higher than those of others. The gap reaches 10 times, which indicates severe abnormalities. Therefore, to eliminate the influence on $\mathrm{I} / \mathrm{O}$ performance from the multiuser and multitask environment, we perform a test for multiple times and choose the average of the execution time as experiment result. Generally speaking, all the following experiments results are the average value of multiple tests.

6.3.2. Evaluation Methodology. The purpose of our experiments is to obtain the performance optimization effect of $A P$ $I O$ in OpenFOAM by comparison of results before and after the optimization. The tests are performed in two dimensions: multiple numbers of processors and multiple write intervals. Our tests start from 4 processors and scale to 1024 processors with a scale coefficient of 2 . Meanwhile, to verify the write interval's effect on I/O performance, we have to get all the benchmarks tested, respectively, with $1 \Delta t, 2 \Delta t, 4 \Delta t, 10 \Delta t$, and none (no snapshot output) write interval conditions.

Our experiments mainly tested three indicators of the performance: the growth of the output file's volume that scales with the number of processors, the original file write overhead without AP-IO optimization, and the optimization effect with $A P-I O$ method. The first two aspects mainly analyze the I/O characteristics of the tree benchmarks, which will be taken as the baseline. The third aspect is to measure the effect of $A P-I O$ optimization directly.

First, to measure the growth of the output volume with the influence of the number of processors, we get the three benchmarks tested under the number of processors ranging from 2 to 1024 and four write interval conditions $(1 \Delta t, 2 \Delta t$, $4 \Delta t$, and $10 \Delta t$ ). Recording the volume of the output file, we will get the characteristics of file volume growth due to the parallel grid partition.

Second, we have to measure the overhead of the periodic snapshot output without AP-IO optimization. Suppose that the execution time under the none write interval condition is $T_{0 \Delta t}$, and the execution time under $n \Delta t$ write interval condition without optimization is $T_{n \Delta t}^{\text {prim }}$, then the file write overhead $T_{\text {file }}^{\text {prim }}$ can be approximated by the following methods as

$$
T_{\text {file }}^{\text {prim }}=T_{n \Delta t}^{\text {prim }}-T_{0 \Delta t}
$$

Finally, the optimization effect of $A P-I O$ is measured with two indicators: one is the optimization ratio to the snapshot output overhead, and the other is the optimization ratio to the total simulation time. We define the total execution time of the simulation with $A P-I O$ under $n \Delta t$ write interval condition as $T_{n \Delta t}^{\mathrm{opt}}$, and then the file write overhead with $A P$ IO optimization $T_{\text {file }}^{\mathrm{opt}}$ can be approximated as $\mathrm{s}$

$$
T_{\text {file }}^{\mathrm{opt}}=T_{n \Delta t}^{\mathrm{opt}}-T_{0 \Delta t}
$$
$\alpha$ is

The optimization ratio of $A P-I O$ to the file write overhead

$$
\alpha=\frac{T_{\text {file }}^{\text {prim }}-T_{\text {file }}^{\text {opt }}}{T_{\text {file }}^{\text {prim }}} .
$$

The optimization ratio of $A P-I O$ to the total simulation time $\beta$ is

$$
\beta=\frac{T_{n \Delta t}^{\mathrm{prim}}-T_{n \Delta t}^{\mathrm{opt}}}{T_{n \Delta t}^{\mathrm{prim}}} .
$$

\subsection{Results and Analysis}

6.4.1. Volume of the Snapshot Output. As shown in Figure 9, they are the total volumes of the three benchmarks' (dambreak, cavity, and pitzDaily) snapshot output tested with the number of processors from 2 to 1024 under different write interval conditions. We can find that the total volume scales with the same rate as the number of processors grows; the rate is about $2 \%$ for dambreak, about $6 \%$ for cavity, and $3 \%$ for pitzDaily. The growth of the volumes is mainly due to the data redundancy in the grid and fields produced by parallel grid domain decompose. Although the growth rate is less than $10 \%$, it still has severe influence on I/O performance, which will be verified and analyzed in Section 6.4.2.

6.4.2. File Write Overhead. To get the baseline of the AP$I O$ optimization, we first have to measure the periodical snapshot output overhead for the entire simulation. Figure 10 shows the three benchmarks' file write overhead.

As mentioned before, we measure the file write overhead indirectly with the difference between the execution time under certain write interval conditions and the none write interval condition. Figures $11(\mathrm{a}), 11(\mathrm{~d})$, and $11(\mathrm{~g})$ are the 


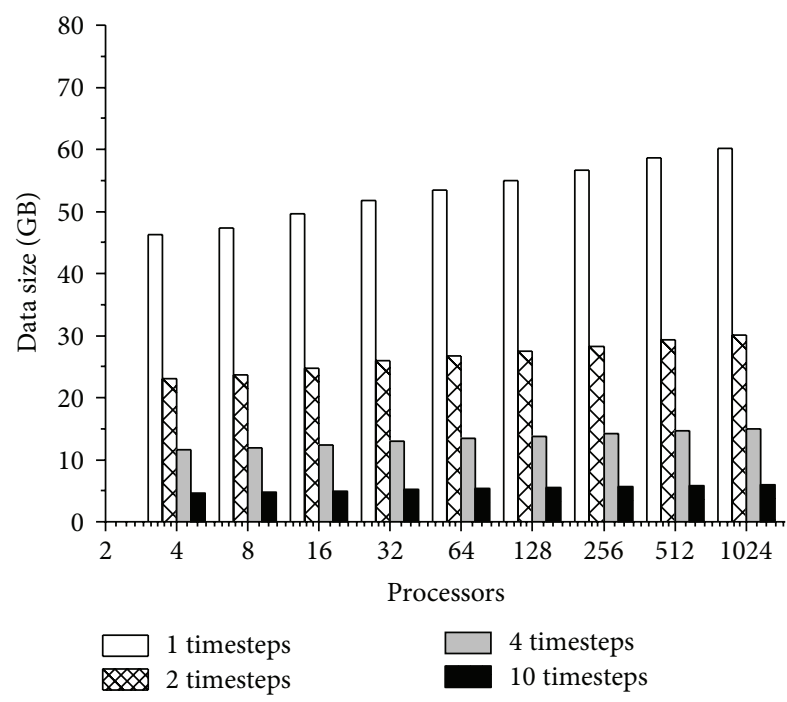

(a) Output data size of dambreak

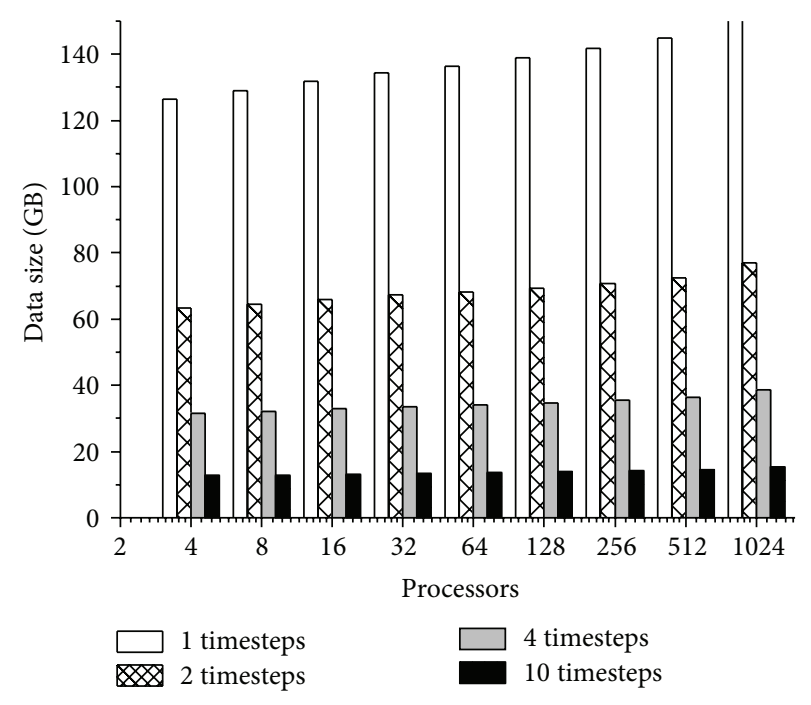

(b) Output data size of cavity

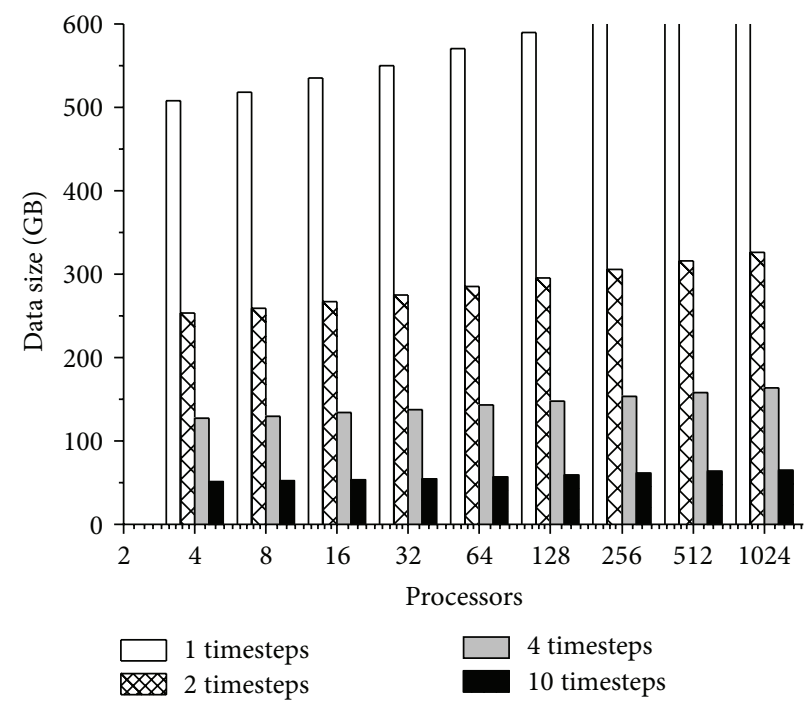

(c) Output data size of pitzDaily

FIGURE 9: CFD application file output volume increases with scale of the number of processors.

execution time of the three benchmarks under the none write interval condition, respectively. It can be found that the performance improves as the number of processors increases, but the scalability is limited at 512 processors.

Figures 11(b), 11(e), and 11(h) show the three benchmarks' file write overhead under different write interval conditions and the number of processors. It can be found that the file write overhead declines with the scale of the number of processors firstly. This phenomenon is mainly due to the fact that the write operations are also performed in parallel with the parallel of processors. On the premise that the I/O channel bandwidth is sufficient, the snapshot output speed scales with the number of processors. But when the number of processors scales to a certain value (64 in our results), the I/O channel bandwidth is not sufficient, and the competition caused by the concurrent write operations makes the write speed decline.
Figures 11(c), 11(f), and 11(i) show how the three benchmarks' proportion of file write overhead in the total simulation time scales with the number of processors. It can be found that although the write file overhead declines with the scale of the number of processors, the proportion of the overhead in the total simulation time ascends. This is due to the fact that the growth of the number of processors makes the file write overhead descend, but its descending speed is far less than that of the calculation time, which makes the proportion of the snapshot output overhead in total simulation time show an ascending tendency. It is notable that the snapshot output overhead portion of dambreak at 1024 processors is lower than that at 512 processors in Figure 10(c), which is due to the rise of calculation time caused by this benchmark's negative speedup at 1024 processors. 

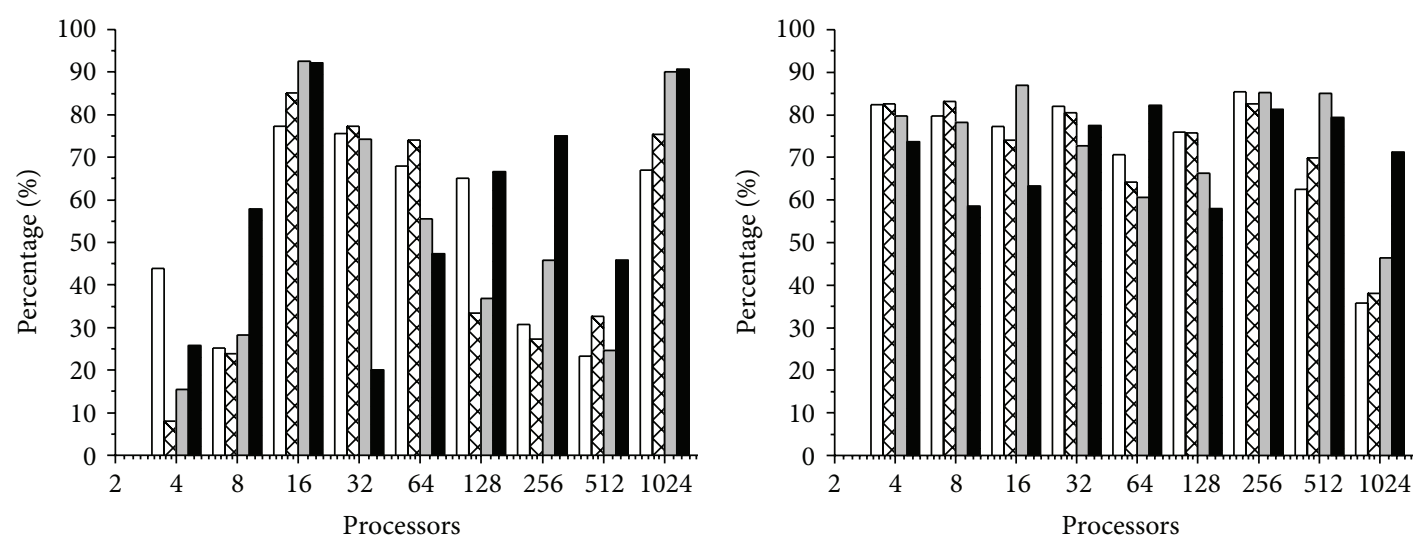

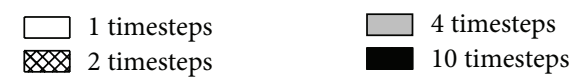

(a) Optimization of write time in dambreak
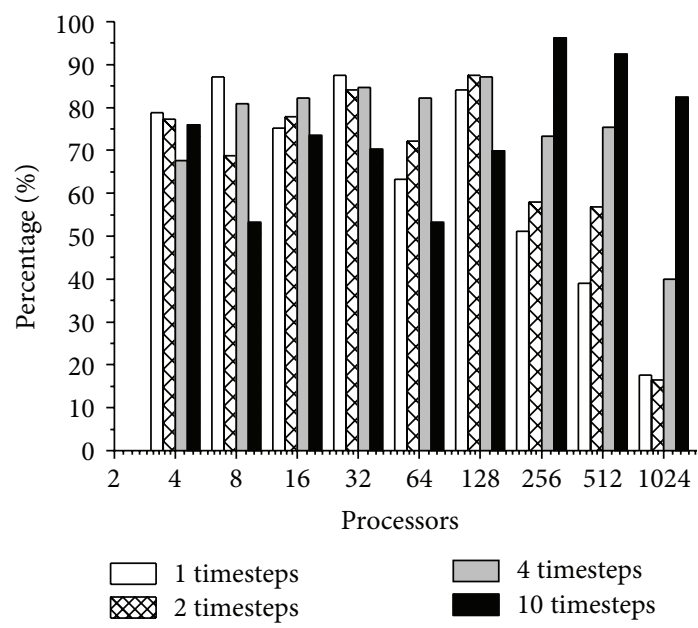

(c) Optimization of write time in pitzDaily
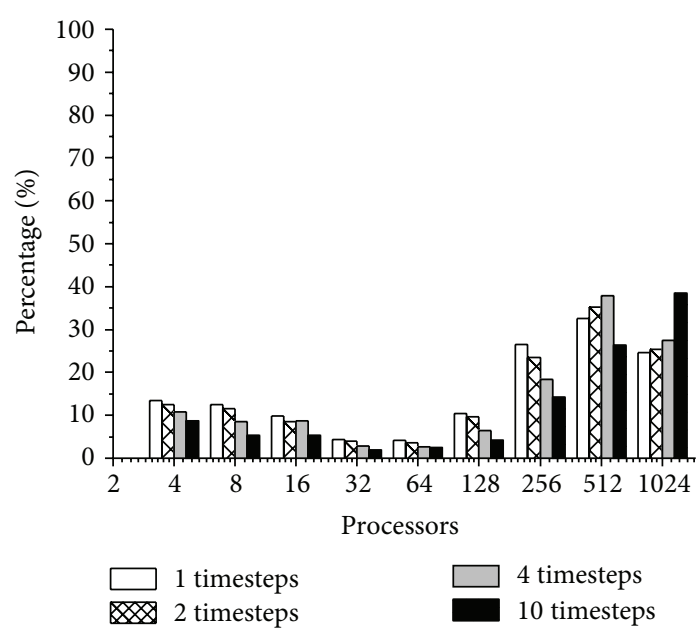

(e) Optimization of total time in cavity

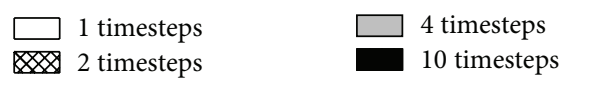

(b) Optimization of write time in cavity

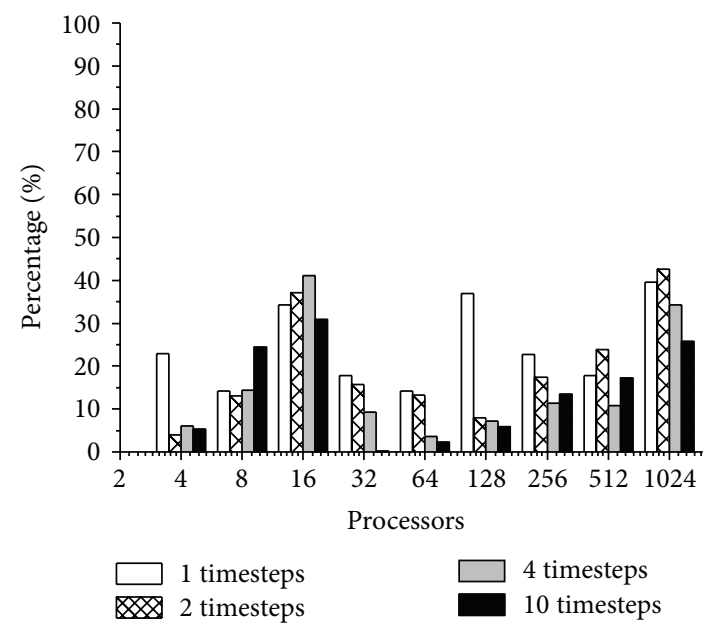

(d) Optimization of total time in dambreak

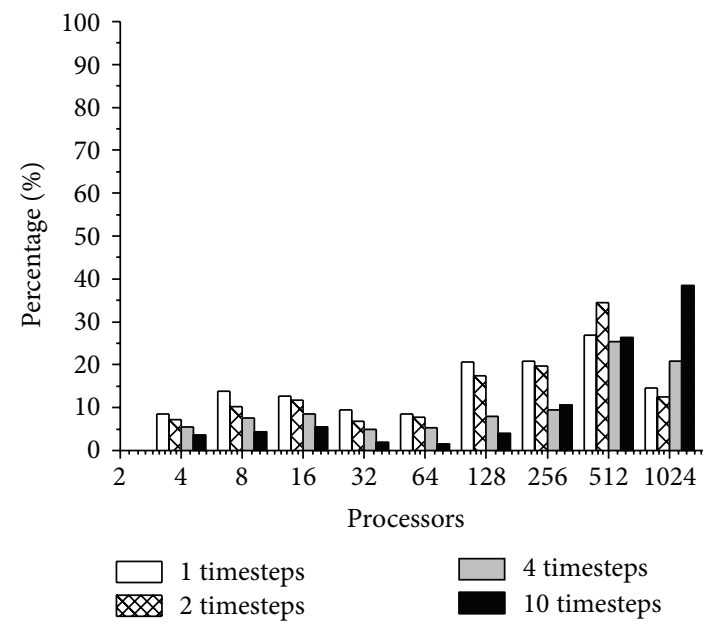

(f) Optimization of total time in pitzDaily

FIGURE 10: Overhead and proportion of periodical file write. 


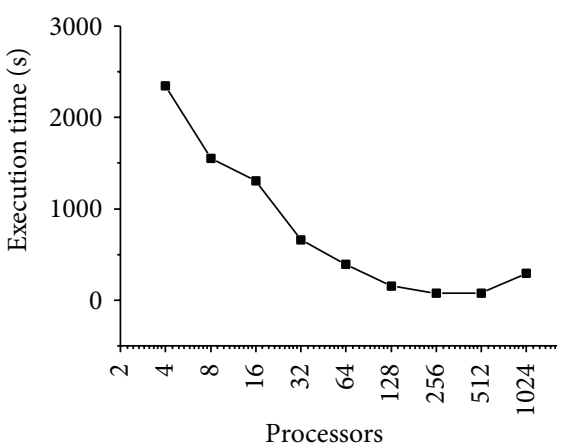

(a) Execution time of dambreak without write

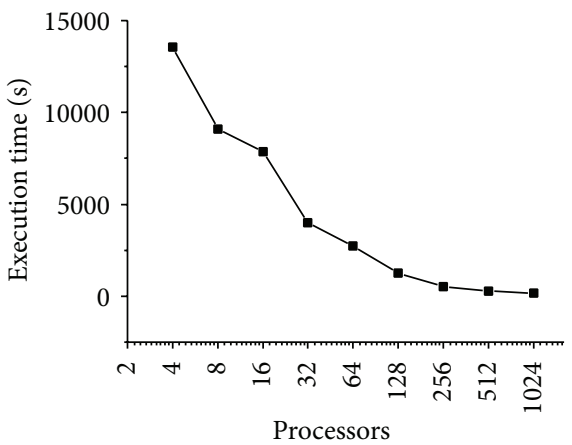

(d) Execution time of cavity without write

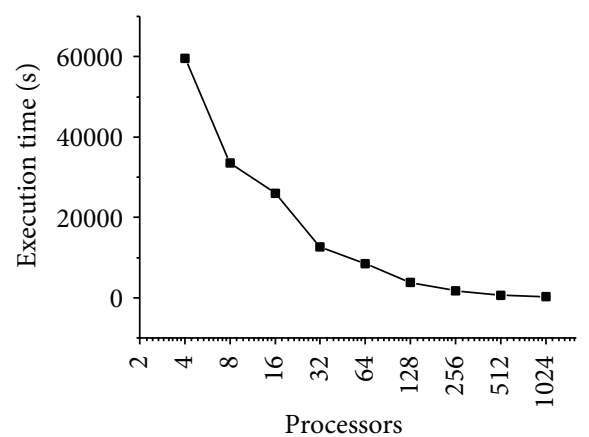

(g) Execution time of pitzDaily without write

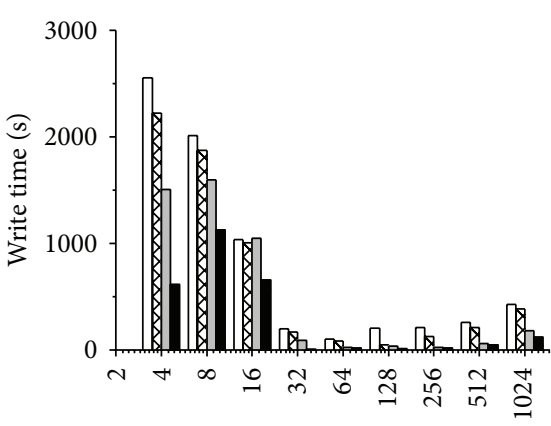

Processors

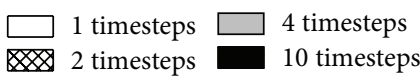

(b) Write time of dambreak

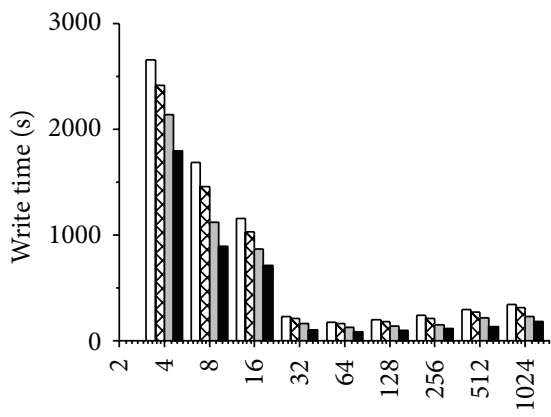

Processors

1 timesteps $\square 4$ timesteps

2 timesteps 10 timesteps

(e) Write time of cavity

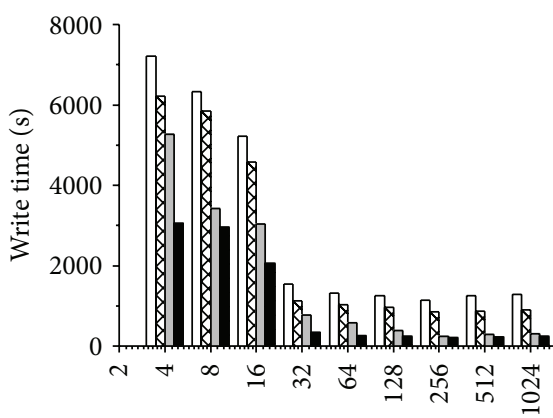

Processors

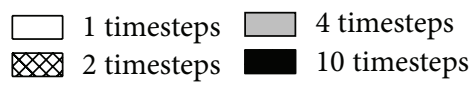

(h) Write time of pitzDaily

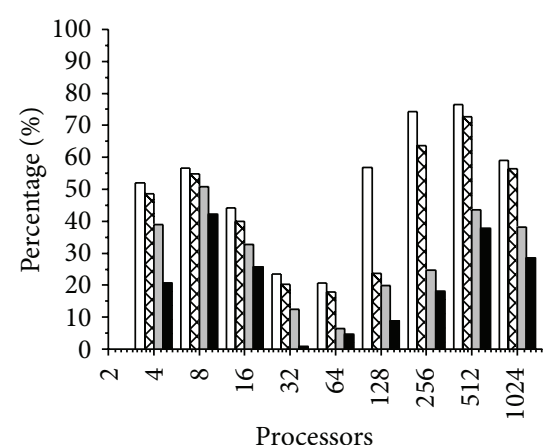

Processors

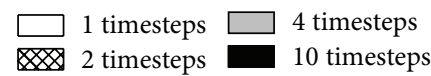

(c) Write time percentage of dambreak

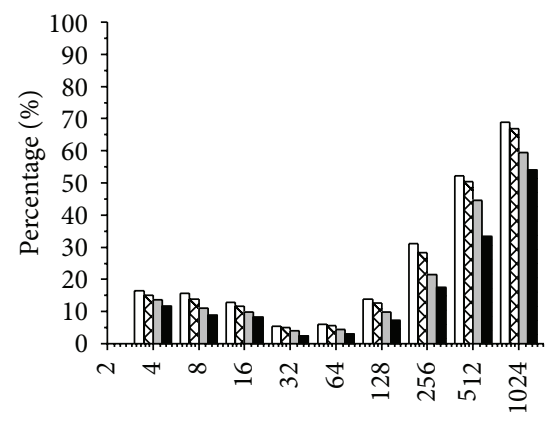

Processors

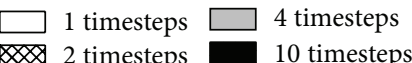

(f) Write time percentage of cavity

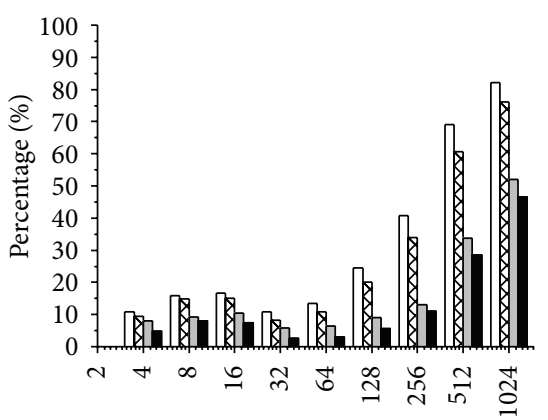

Processors

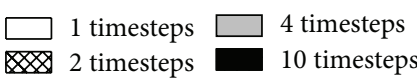

(i) Write time percentage of pitzDaily

FIGURE 11: Optimization effect of AP-IO.

6.4.3. Optimization Effect of AP-IO. Figure 11 shows the optimization effect of $A P-I O$, which is measured with the optimizing effect on the snapshot output overhead and the total simulation time, respectively. As shown in Figures 10(a)10 (c), the optimization ratio of snapshot output overhead can reach more than $50 \%$ in average and $90 \%$ in the best case.

As shown in Figures 11(d)-11(f), the optimization ratio of the total simulation time can reach to $10 \%$ with $A P$ $I O$ on average. However, as the snapshot output overhead is hidden by the calculation operation, this optimization ratio of the total simulation time cannot exceed $50 \%$. The trends in the figure show that $A P-I O$ is more effective for the simulations under the intensive write interval condition. The optimization effect of the total simulation time tends to decrease when the number of processors is smaller than 64 . However, when the number of processors is more than 64 , the optimization effect of the total simulation time tends to increase. This phenomenon agrees with the trends of the file write overhead ratio in the total simulation time shown in Figure 10. 
The experimental results show that $A P-I O$ can well optimize the periodic snapshot output in CFD simulation and gains better optimization effect in massively parallel simulations.

\section{Conclusions}

We have proposed an AP-IO optimization method based on the features analysis of CFD simulation for the problem of periodic large volume snapshot output in CFD simulation. We design the AP-IO framework for CFD application by combining the compiler-directed technology and the application library. We implement AP-IO with multithreading technology in the open source CFD software OpenFOAM. Experimental results demonstrate that our AP-IO optimization technique can achieve a good optimization effect for the periodical snapshot output in CFD simulation, which can reduce $10 \%$ of the total execution time on average.

\section{Conflict of Interests}

The authors declare that there is no conflict of interests regarding the publication of this paper.

\section{Acknowledgments}

We are pleased to acknowledge the National Natural Science Foundation of China (Grant nos. 61221491, 61303071, and 61120106005), Fund (no. 134200026) from Guangzhou Science and Information Technology Bureau, and Fund of State Key Laboratory of High Performance Computing (no. 201303-01).

\section{References}

[1] D. Roose and R. Vandriessche, Eds., Parallel Computers and Parallel Algorithms for CFD: An Introduction, 1995.

[2] T. Norton and D.-W. Sun, "Computational fluid dynamics (CFD) - an effective and efficient design and analysis tool for the food industry: a review," Trends in Food Science and Technology, vol. 17, no. 11, pp. 600-620, 2006.

[3] A. Iserles, "Parallel computational fluid dynamics: implementation and results," Journal of Fluid Mechanics, vol. 254, pp. 722723, 1993.

[4] J. No, R. Thakur, and A. Choudhary, "High-performance scientific data management system," Journal of Parallel and Distributed Computing, vol. 63, no. 4, pp. 434-447, 2003.

[5] H. Mickler, A. Knüpfer, M. Kluge, M. S. Müller, and W. E. Nagel, "Trace-based analysis and optimization for the semtex CFD application-hidden remote memory accesses and I/O performance," in Euro-Par 2008 Workshops-Parallel Processing, pp. 295-304, Springer, Berlin, Germany, 2009.

[6] G. P. Lim, F. Yang, T. Chu, P. Chen et al., "Ibuprofen suppresses plaque pathology and inflammation in a mouse model for Alzheimer's disease," The Journal of Neuroscience, vol. 20, no. 15, pp. 5709-5714, 2000.

[7] X. Ma, M. Winslett, J. Lee, and S. Yu, "Improving MPI-IO output performance with active buffering plus threads," in Proceedings of the 17th International Symposium on Parallel and Distributed
Processing (IPDPS '03), pp. 68.2-68.5, IEEE Computer Society, Washington, DC, USA, 2003.

[8] X. Ma, J. Lee, and M. Winslett, "High-level buffering for hiding periodic output cost in scientific simulations," IEEE Transactions on Parallel and Distributed Systems, vol. 17, no. 3, pp. 193-204, 2006.

[9] D. J. Mavriplis and S. Pirzadeh, "Large-scale parallel unstructured mesh computations for 3D high-lift analysis," Journal of Aircraft, vol. 36, no. 6, pp. 987-998, 1999.

[10] W. D. Gropp, D. K. Kaushik, D. E. Keyes, and B. F. Smith, "Highperformance parallel implicit CFD," Parallel Computing, vol. 27, no. 4, pp. 337-362, 2001.

[11] S. Iyer and P. Druschel, "Anticipatory scheduling: a disk scheduling framework to overcome deceptive idleness in synchronous I/O," SIGOPS: Operating Systems Review, vol. 35, no. 5, pp. 117-130, 2001.

[12] A. Acharya, M. Uysal, R. Bennett et al., "Tuning the performance of I/O-intensive parallel applications," in Proceedings of the 4th Annual Workshop on I/O in Parallel and Distributed Systems (IOPADS '96), pp. 15-27, ACM, May 1996.

[13] N. Ali, P. Carns, K. Iskra et al., "Scalable I/O forwarding framework for high-performance computing systems," in Proceedings of the IEEE International Conference on Cluster Computing and Workshops (CLUSTER '09), New Orleans, La, USA, September 2009.

[14] http://www.top500.org/.

[15] J. Dongarra, Visit to the National University for Defense Technology, 2013. 

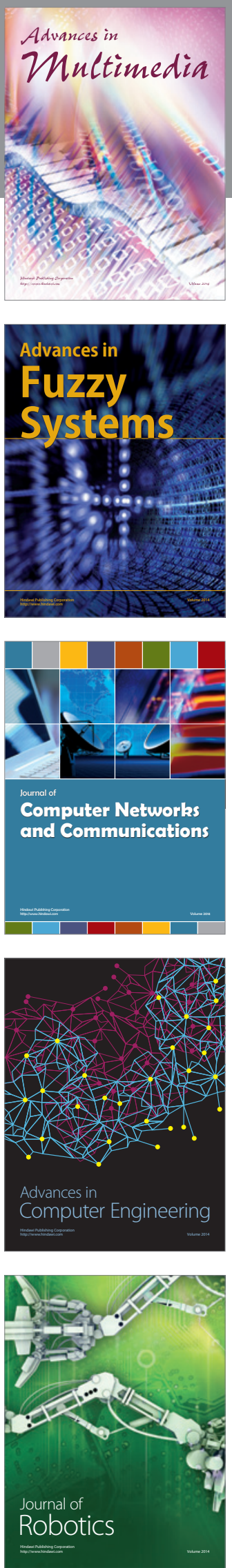

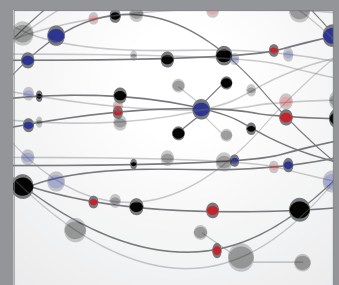

The Scientific World Journal
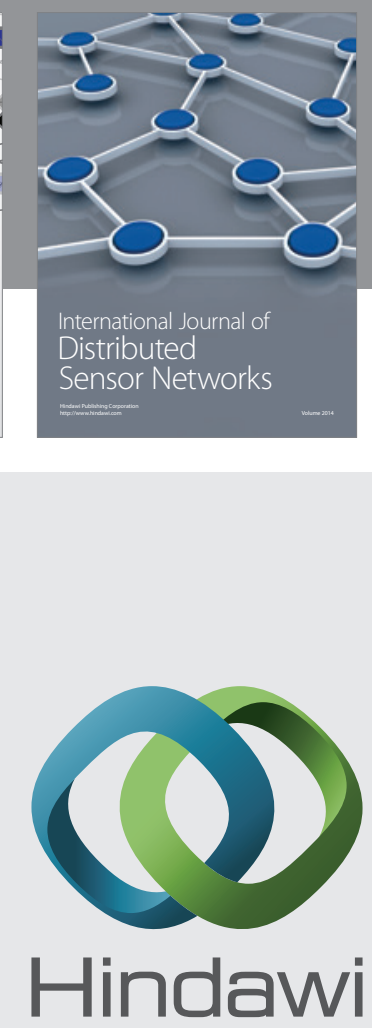

Submit your manuscripts at

http://www.hindawi.com
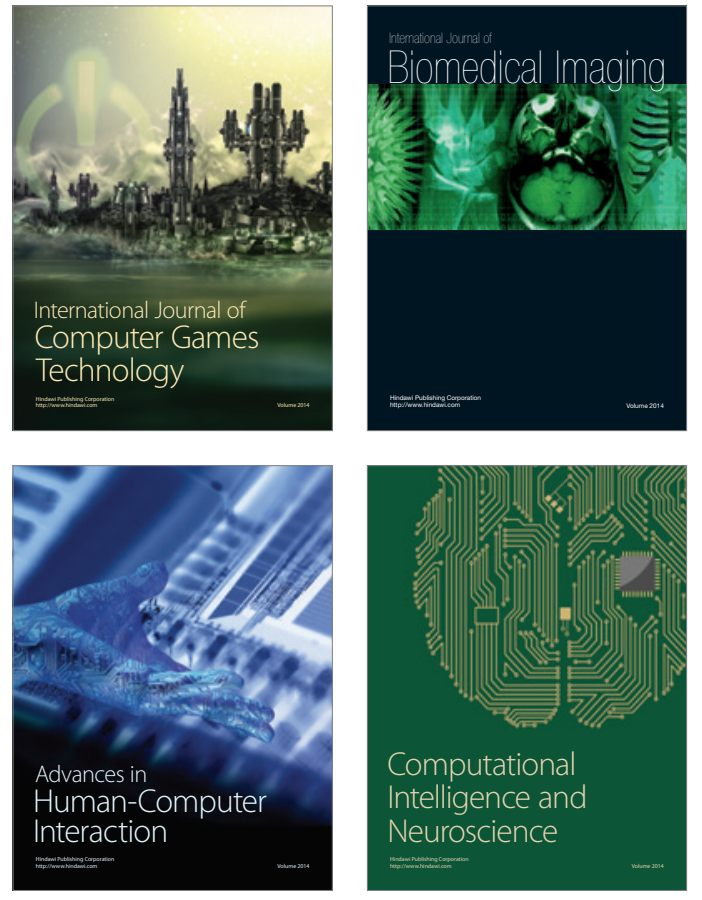
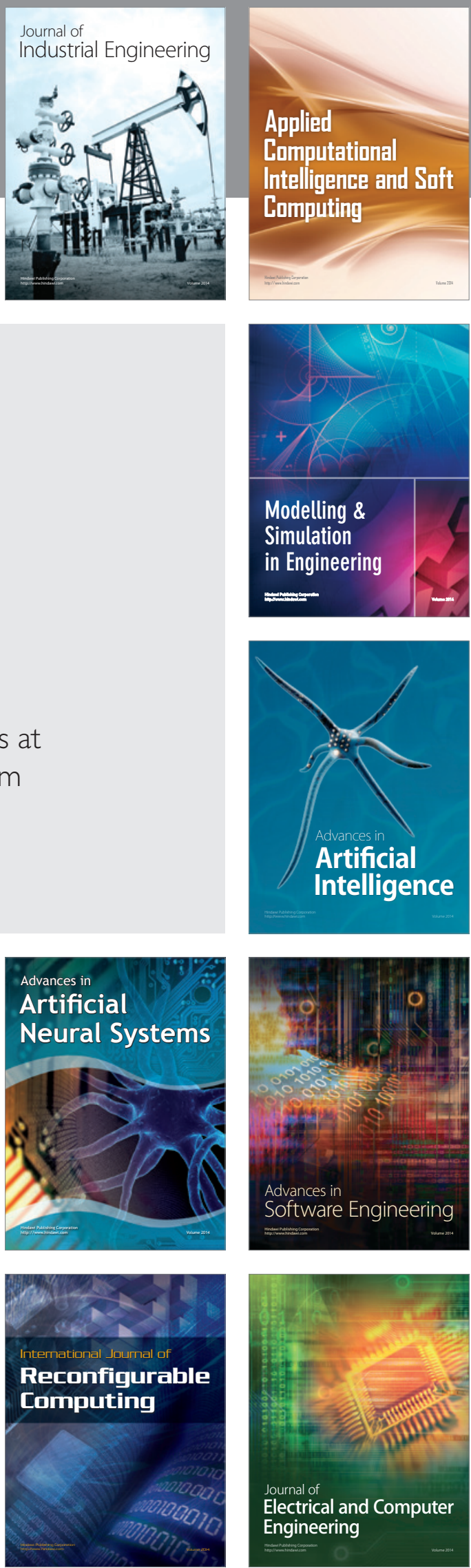